\title{
Physics of Ionized Gases
}

\section{Individual Ordinary} Members

\author{
Key to table opposite \\ $1=$ Total Members \\ 2 = Astronomy and Astrophysics Div. \\ 3 = Solar Physics Section \\ $4=$ Atomic Physics Division \\ $5=$ Atomic Spectroscopy Sect. (EGAS) \\ $6=$ Chemical Physics Section \\ $7=$ Electronic and Atomic Collis. Sect. \\ $8=$ Molecular Physics Section \\ 9 = Computational Physics Group \\ $10=$ Condensed Matter Division \\ $11=$ Low Temperature Section \\ 12 = Macromolecular Physics Section \\ 13 = Magnetism Section \\ $14=$ Metals Section \\ 15 = Semiconductors and Insulat. Sect. \\ $16=$ Surfaces and Interfaces Section \\ $17=$ High Energy and Part. Physics Div. \\ $18=$ Nuclear Physics Division \\ $19=$ Plasma Physics Division \\ $20=$ Quantum Electronics Division
} they involv statistical analysis which are, on the whole, understood. Since now a great deal of information exists, obtained both from beam experiments and from quantum theory, about the collision cross-sections, one might imagine that the problem of the ionized gas was solved, and that quantitative predictions would be possible from first principles. The physicists who attended the 3rd. European Study Conference on Atomic and Molecular Processes in Ionized Gases (ESCAMPIG) at Bratislava, 24-26 August, are well aware how far they are from such a state. Even fully ionized plasmas, consisting of protons and electrons, are full of complications arising from impurities and surfaces.

Neither plasma nor laser physicists are centrally concerned with atomic and molecular processes whilst atomic collision physicists are rather shy of plasmas. The ESCAMPIG meetings have thus filled a gap, first in Western physics and now with the Bratislava meeting in Eastern Europe as well. Of the invited papers and progress reports, $60 \%$ came from the East.

It used to be the case that electron collision processes dominated these discussions. The emphasis was on plasma spectroscopy and on the important part played by collisional radiative recombination, such as occurs in dense plasmas and in the solar corona. The methods first proposed by David Bates, in Belfast, were fully exploited, and at this meeting, the discussions in radiative diffusion were continued by L.M. Biberman. But now the interest is much more in heavy particle collisions - charge transfer, associative detachment, excitation exchange, quenching, ion-molecule protages. These are also the collision processes now coming into their own in astrophysics, not only in the aeronomy of planetary atmospheres, but in the case of ion-molecule processes, in the problem of the origin of molecules in interstellar space.

The most interesting part of this meeting was the emphasis on the role played by these heavy particle collisions, in laser pumping (N.N. Sobolev, C.B. Collins), in alkali plasmas (J. Berlande), in mobility and conduction (B.M. Smirnov), and in cataphoresis (L.M. Chanin). The flowing afterglow has become an important, although expensive, technique for studying rate processes, and now the initiation of the ion flow situation by injection of mass-separated ion beams has been shown by David Smith to be advantageous.

It is true that the physics of the ion-molecule processes contains much interesting semi-classical and quantum theory, in the way of computer dynamics, curve-crossings and Stückelberg oscillations; but the effort involved in making good calculations of many of these cross-sections is orders of magnitude greater than the effort involved in measuring them. Therefore the rate process apparatus, whether it be afterglow, drift tube, or modulated plasma, will be around for a long time to come. Such apparatus is an investment, which should perhaps be made more widely in European laboratories. Our hosts for this meeting, at the Comenius University, in Bratislava, are typical of a laboratory building up its facilities in this field. They provided us with an excellent meeting, a challenge to the organizers for 1978, when the meeting will be held in Britain.

J.B. Hasted
Individual Ordinary Members are reminded that four of their nine delegates to Council are due for replacement in 1977 and nominations to these positions must be received by the Secretariat before the end of this year.

\section{Condensed Matter Div.}

An EPS sponsored International Conference on Lattice Dynamics will be held 5-10 September, 1977 in Paris, under the auspices of IUPAP and other organizations. Although aspects of lattice dynamics and phonons have been treated at various international conferences in recent years, the forthcoming international conference in Paris will be the first general conference, since the one held in Copenhagen in 1963, in which "Lattice Dynamics" is the central theme.

The major focus will be on basic phonon properties, non-linear lattice dynamics, lattice dynamics of imperfect crystals and lattice instabilities, themes which are basic to the subject as a whole. The conference will also focus on such topics as lattice dynamics of low dimensional systems and of superionic conductors, which are of high current interest. The choice of topics for the conference will take into consideration the fact that an International Conference on Ferroelectricity will be held in Leningrad (18-23 September, 1977) and that an International Conference on Neutron Scattering will be held in Moscow (12-16 September, 1977).

Further information can be abtained from Prof. M. Balkanski, Laboratoire de Physique des Solides, Université Pierre et Marie Curie, 4, Place Jussieu, F-75230 Paris 05. 